\title{
A METHOD OF PARAMETERS IDENTIFICATION FOR THE COUPLED DRY FRICTION MODEL FOR PNEUMATIC TIRES
}

\author{
ALEXEY A. KIREENKOV ${ }^{1,2}$, SERGEY I. ZHAVORONOK ${ }^{3}$ \\ ${ }^{1}$ Moscow Institute of Physics and Technology (State University), Institutskiy per. 9, Dolgoprudny, \\ Moscow Region, 141701, Russia, kireenkov.aa@mipt.ru \\ ${ }^{2}$ Ishlinsky Institute for Problems in Mechanics of Russian Academy of Sciences (IPMech RAS), \\ Prospekt Vernadskogo 101, korp.1, 119526, Moscow, Russia, kireenk@ipmnet.ru
}

${ }^{3}$ Institute of Applied Mechanics of Russian Academy of Sciences (IAM RAS), 125040, Leningradskiy prospect 7, Moscow, Russia, zhavoronok@iam.ras.ru

Key words: Combined Dry Friction, Coupled Dry Friction, Pneumatic Tire.

\begin{abstract}
The theory of multi-component dry friction accounting for the coupled kinematics of relative motion in finite contact spots is applied to the modelling of pneumatic tires. The analytical models of the combined dry friction accounting for the anisotropy of the dry friction factors as well as the distribution of the contact pressure close to the real one are introduced. The exact integral relationships between the dry friction force and torque and the generalized velocities, i. e. the speed of sliding and angular velocity of spinning, appears as a result of integration over the contact spot and are too complex to be implemented analytically into the engineering practice. The proposed approximate models are based on the fractional approximations and could be interpreted as rheological models with low number of constitutive constants. These constants could be identified after the solution of some specific inverse problems with input data given by the diagrams of the dry friction forces and torque obtained as a result of simple physical tests. Here these inverse problem are formulated and solved using the perturbed benchmark solutions of the corresponding direct problems with the factors obtained after the numerical solution of the contact problem for the heterogeneous pressurized tire. The possibility of stable solution for the inverse problems on the basis of the proposed approach is shown. The presented models as well as the method if constitutive constants identification could be applied for more detailed investigation of unsteady rolling regimes of pneumatics which are characterized by the non-vanishing sliding and spin.
\end{abstract}

\section{INTRODUCTION}

The "shimmy", i.e. the high-amplitude coupled lateral and torsional vibrations of controllable nose landing gears of aircrafts is a well-studied phenomenon [1,2]. At the same time the shimmy-like oscillations of rigidly fixed main landing gears is not so widely known and moreover not investigated in details in spite of the fact that they were often observed during operation of some modern aircrafts, moreover such oscillations resulted destructions of torque links of gears and even caused hard failures of aircrafts' frames in some registered cases. The main specific features of such instable rolling regimes are the following ones: 
- the oscillations appear at initial stages of landings shortly after touchdown (i.e. during non-steady wheels rolling with longitudinal sliding);

- the significant sliding of wheels at unstable regimes is proved by tires' tracks at runaways surfaces.

Thus, the classical shimmy model based on the non-holonomic constraint following from the assumption of vanishing slip and spin of a wheel $[1,2]$ fails evidently, therefore a qualitatively hew theory is required. Such a model should take into account as well dry friction effects as deforming of pneumatic tires. A first attempt of accounting for the finite dimension of the area of contact of a wheel and a road and, consequently, for the coupling dynamic effects of the combined kinematics of relative motion of interacting wheel of an landing gear and a runaway surface under the condition of intensive sliding was proposed in [3] where the dynamics of the asymmetric one-wheel main landing gear was studied in. It was shown that the shimmy phenomenon can be provoked only by dry friction forces under the conditions of combined kinematics. The coupled dry friction theory was further developed in several works [4-14]. In spite of efficiency of the approach [3] it cannot be implemented in the engineering practice since the Hertzian distribution of the contact pressure has almost nothing to do with the real one observed for severely deformed pressurized pneumatic tires (except very small loads). The first improved model accounting for the random distribution of the normal contact stresses inside of contact patches was introduced in [15]. Thus, the second step required to adopt the theory of coupled dry friction to the problems of rolling stability for pneumatic tires consists in the use of accurate approximations of the contact pressure; indeed this distribution impac rolling stability domai distribution could be ob [16] or using geometric Let us note that the
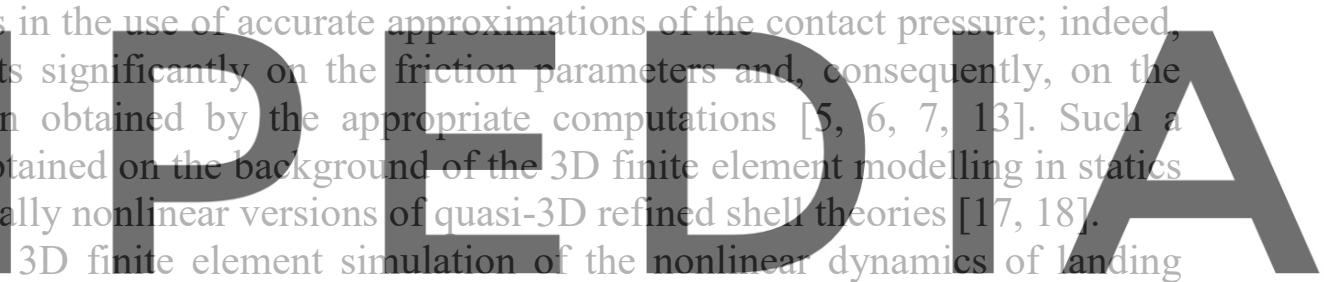

gears remains too resource consumptive even today to be implemented into the engineering

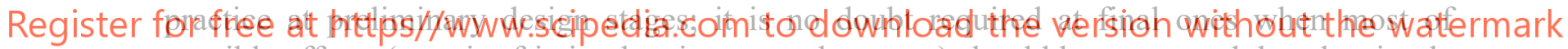
possible effects (e.g. tire friction heating, mass loss, etc.) should be accounted, but the simple models with few degrees of freedom like [1] are required to predict which effects have to be studied before the detailed quantitative dynamic analysis will be performed [19]. Thus, the development of a simplified shimmy model accounting as well for the dry friction with combined kinematics as for the deformed state is a topical problem. Indeed, some attempts of use of of the coupled dry friction theory with quasi-rigid wheel model and Hertz's solution for the contact pressure allowed not only to calculate the dry friction forces and torque but to estimate also the effects of the dry friction on the dynamics as it was made in [4,5,7-10]. One of first attempts of accounting for the contact pressure distribution close to real ones was made in [7]; the finite element solution was approximated by the Legendre polynomials, then the model coefficients were computed analytically. The coefficients definition remained the drawback limiting the practical use of the model [7]. The technique of experimental definition of these coefficients was presented in [19]. The validation procedure proposed below consists in two main stages: at the first one the coefficients are calculated using analytical formulae $[4,5,7,9]$ with aid of numerical simulation of the contact pressure distribution, then these coefficients are defined from the numerical dependence for dry friction torque and force components on the slip and spin velocities. 


\section{GENERAL MODEL OF THE COUPLED DRY FRICTION}

For a general case, let us consider the orthotropic dry friction given by the tensor $\mathbf{f}$ defined within its principal components $f$ and $\kappa f$, and the Cartesian frame $O x y$ attached to the centroid of the area $S$ of contact of a wheel and a road; we consider below only axially symmetric contact areas having the average radius $R$; the appropriate base vectors $\mathbf{e}_{1}$ and $\mathbf{e}_{2}$ are collinear to the principal axes of $\mathbf{f}$. Finally, let us assume the static contact pressure distribution, $\sigma_{0}(x, y)=\sigma_{0}( \pm x, \pm y)$, be symmetric with respect to the center $O$ of the area $S$.

Let the motion of a wheel be defined by the longitudinal velocity $\mathbf{v}_{0}=v \mathbf{e}_{1}$ (i.e. along the axis $O \xi$ of the global rest frame; for more details, see [5] and [7]), the angular velocity of rolling $\boldsymbol{\omega}_{\tau}=-\Omega_{r} \mathbf{e}_{2}$, and the velocity of spinning $\omega$. The presence of a thread of any real tire is modeled by the different dry friction factors $f$ along the tread and $\kappa f$ across it. The rolling friction is defined by the vector d representing the shift of the gravity center of the contact area $S$ with respect to its geometric center $O$, therefore the deformed contact pressure distribution could be introduced as $\sigma(x, y)=\sigma_{0}(x, y)\left(1+k_{r} x+k_{y} y\right)$ [14] where $k_{r}$ and $k_{y}$ are projections of the vector $\mathbf{d}$ on axes $x$ and $y$. We could define hence the friction-induced tangent strain at arbitrary point $M \in S$ as follows:
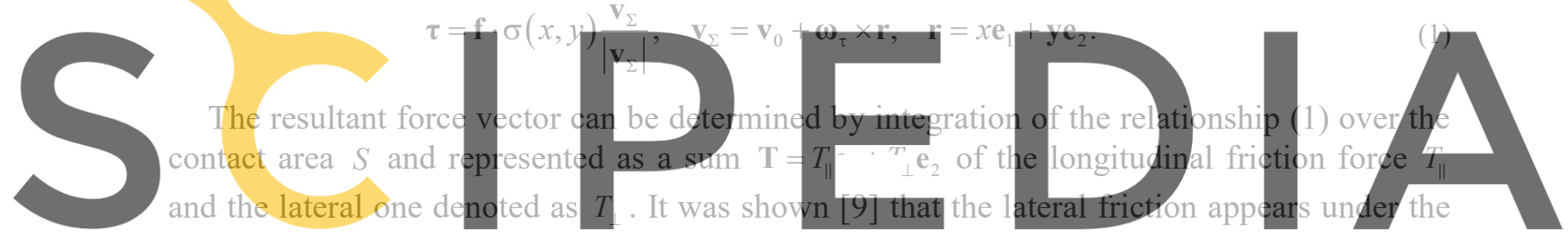

conditions of simultaneous slip and spin due to the coupling effects. The orthogonal

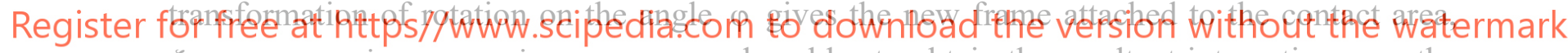
$\xi=x \cos \varphi-y \sin \varphi, \eta=x \sin \varphi+y \cos \varphi$, and enables to obtain the resultant integration over the

fixed domain [9], therefore the appropriate expressions can be simplified keeping in mind that integrals will be zero for symmetric integration domains and odd integrand function by one of the arguments. As a result, for $\kappa=1$ we obtain for the friction force and torque

$$
\begin{gathered}
F_{\|}=\tau \iint_{G} \frac{\sigma_{0}\left(1+k_{x} x+k_{y} y\right)(v-\omega(x \sin \varphi+y \cos \varphi)) d x d y}{\sqrt{v^{2}-2 v \omega(x \sin \varphi+y \cos \varphi)+\omega^{2}\left(x^{2}+y^{2}\right)}} \\
M_{v}=f \iint_{G} \frac{\sigma_{0} \omega\left(x^{2}+y^{2}\right)-v(x \sin \varphi+y \cos \varphi) d x d y}{\sqrt{v^{2}-2 v \omega(x \sin \varphi+y \cos \varphi)+\omega^{2}\left(x^{2}+y^{2}\right)}} \\
F_{\perp}=f \iint_{G} \frac{\sigma_{0} \omega(x \cos \varphi-y \sin \varphi) d x d y}{\sqrt{v^{2}-2 v \omega(x \sin \varphi+y \cos \varphi)+\omega^{2}\left(x^{2}+y^{2}\right)}}
\end{gathered}
$$

The model defined by integral formulae (2-4) is interpreted as "exact" one. 


\section{APPROXIMATE MODEL OF THE COUPLED DRY FRICTION FOR A TIRE}

The formulae approximating (2-4) for the longitudinal dry friction force, the lateral dry friction force, and finally for the dry friction torque could be written as follows [7, 19]:

$$
F_{\|} \quad \frac{F_{0} v}{v v^{2}+a u^{2}}, \quad F_{\perp}=\frac{k_{x} M_{0} u}{2 \sqrt{u^{2}+\frac{1}{4} m v^{2}}}, \quad M_{C}=\frac{M_{0} u}{\sqrt{u^{2}+m v^{2}}}
$$

Here the following factors are introduced:

$$
\begin{gathered}
N=2 \pi R^{2} A^{1}, \quad F_{0}=2 \pi f R^{2} A^{1}, \quad M_{0}=2 \pi f R^{3} A^{2} \\
\frac{1}{\sqrt{a}}=\frac{1}{F_{0}} \pi f R^{2} A^{0}=\frac{1}{2} \frac{A^{0}}{A^{1}} ; \quad \frac{1}{\sqrt{m}}=\frac{1}{M_{0}} \pi f R^{3} A^{3}=\frac{1}{2} \frac{A^{3}}{A^{2}} . \\
A^{m}=\int_{0}^{1} \sigma_{0}(\rho) \rho^{m} d \rho, \quad \rho=\frac{r}{R} .
\end{gathered}
$$

\section{CONTACT PRESSURE IN THE SPOT OF TIRE-ROAD CONTACT}

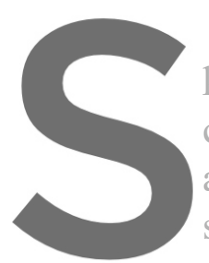

Let us consider a typi

$\mathrm{kPa}$ and loaded by a ver

contact spot diameter;

authors of [14] was pe

spot diameter) correspo
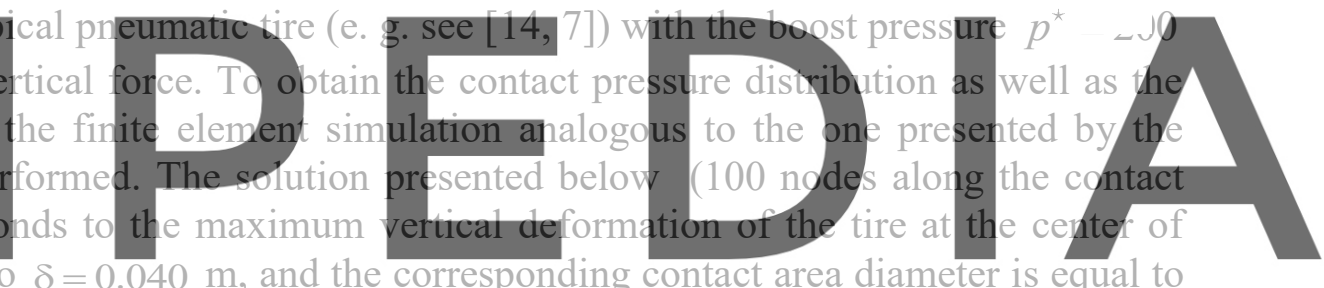

Register $R=0.087 \mathrm{~m}$. The numerically obtained distribution of the dimensionless. contact pressure $p=P Y p^{*}$ is strictly heterogeneous with local maxima near the contact spot boundary of and

the minimum near its center (Figure 1).

To implement the approximate model of the dry coupled friction the analytical interpolation of this pressure distribution is required. Accounting for the symmetry of the contact pressure distribution, let us use the following formula:

$$
p=\sum_{k=0}^{N} a_{k} \cos (\pi k \omega x), \quad x \in[-1,1], \quad \omega \in \mathbb{R} \quad \mathbb{N}
$$

The seventh order approximation (i. e. $N=7$ ) is used below; the corresponding coefficients are shown in the Table 1.

Table 1: Coefficients of the trigonometric approximation of the numerical solution for the contact pressure $\omega$

\begin{tabular}{ccccccccc}
\hline$\omega$ & $a_{0}$ & $a_{1}$ & $a_{2}$ & $a_{3}$ & $a_{4}$ & $a_{5}$ & $a_{6}$ & $a_{7}$ \\
\hline 0.848 & 0.685 & 0.398 & -0.831 & 0.230 & 0.161 & -0.027 & -0.133 & 0.101 \\
\hline
\end{tabular}


The approximated pressure is shown on the Figure 1 by the solid line. One could conclude that the approximation (8) with the coefficients given by the Table 1 offers a good approximation of the numerical solution; it seems to be better than the polynomial interpolation used in [7].

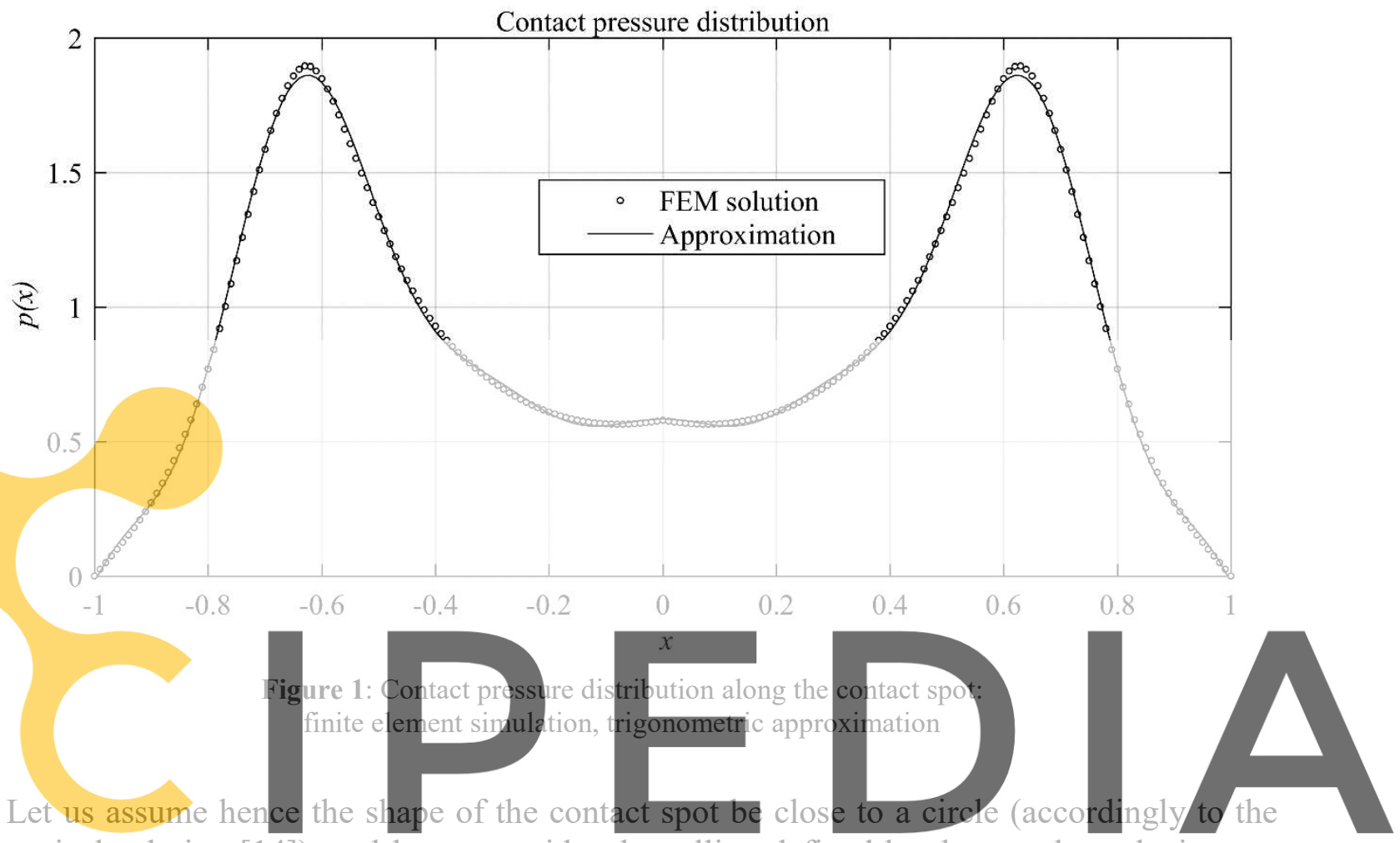

numerical solution [14]), and let us consider the rolling defined by the angular velocity $\omega_{y}$

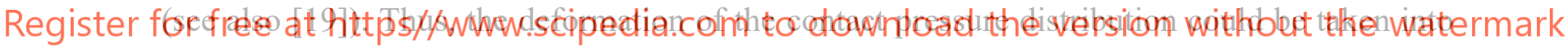
account by introducing the rolling correction factor $k_{x}$ :

$$
p_{\Sigma}=p_{0}(r, \theta)\left(1+k_{x} x\left|\omega_{y}\right|^{-1} \omega_{y}\right)
$$

Here $x$ is the dimensionless coordinate along the contact spot while $k_{x}$ is the value of the shift of the gravity center of the contact pressure distribution due to the rolling.

Let us consider hence the contact pressure distribution corresponding to the steady-state rolling along the $O x$ axis. The numerically obtained solution is shown below on the Figure 2 . The following formulae (see [19]) could be used for the rolling correction factor:

$$
\begin{gathered}
k_{x}=R \frac{s_{x}}{s}, \quad s=\pi R \int_{0}^{1} \sigma_{v}(r, \theta) r^{3} d r, \quad s_{x}=\frac{s_{x}^{1}}{s_{x}^{2}}, \\
s_{x}^{1}=R \int_{0}^{2 \pi} \int_{0}^{1} \sigma_{v}(r, \theta) r^{2} \cos \theta d r d \theta, \quad s_{x}^{2}=R \int_{0}^{2 \pi} \int_{0}^{1} \sigma_{v}(r, \theta) r d r d \theta .
\end{gathered}
$$




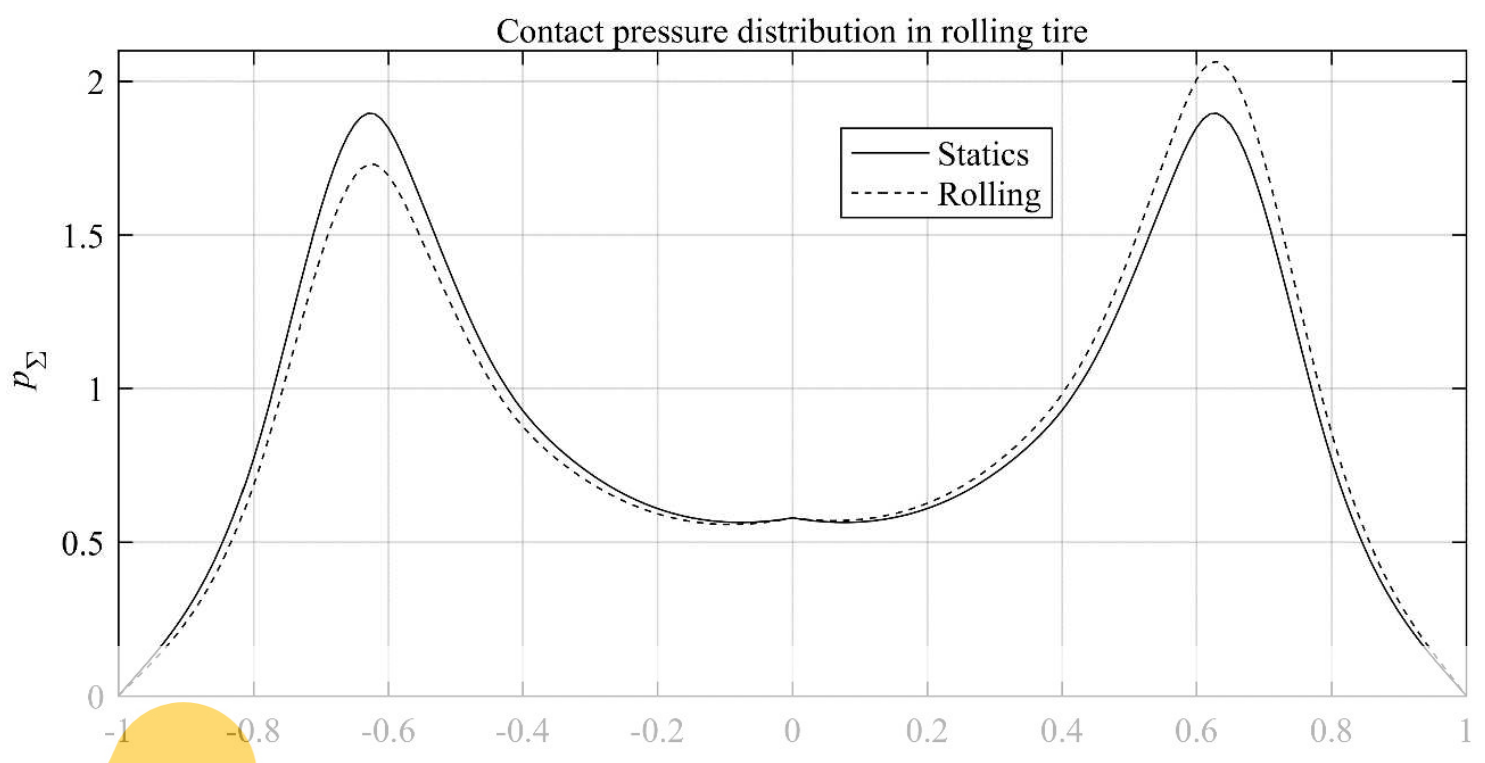

Figure 2: Contact pressure distribution along the contact spot:

finite element simulation, trigonometric approximation

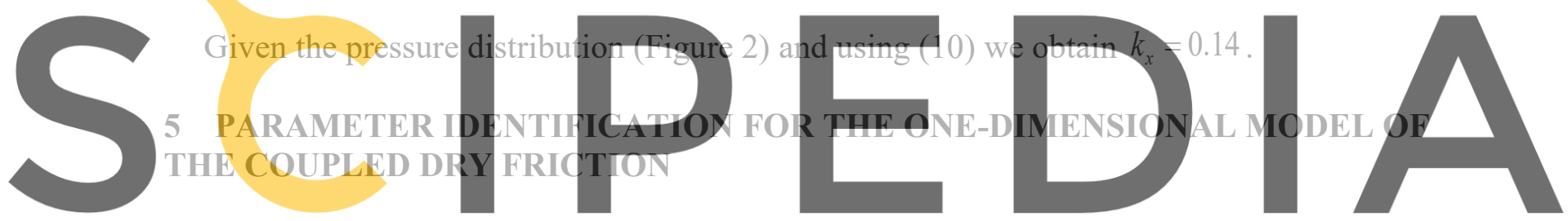

The factors given by (5-7) could be computed using the approximation (8) analytically:

Register for free at https//WwW.scipedia.com to download the version without the watermark

$$
\begin{aligned}
A^{m}= & \frac{1}{m+1} \sum_{k=0}^{N} a_{k}\left\{-k \omega(k \omega)^{-m-5 / 2}\left(k \omega \pi^{-1 / 2-m} \cos (\pi k \omega)-\pi^{-m-3 / 2} \sin (\pi k \omega)\right) S_{m+3 / 2,1 / 2}(\pi k \omega)+\right. \\
& \left.+\pi^{-1 / 2-m}(k \omega)^{-m-3 / 2} S_{m+1 / 2,3 / 2}(\pi k \omega) \sin (\pi k \omega) k m \omega+\cos (\pi k \omega)\right\},
\end{aligned}
$$

here $s_{\mu, v}$ are Lommel's functions. Given boost pressure $p^{\star} \quad-J 0 \mathrm{kPa}$ and accounting for the formula (11), we obtain the following factors values:

Table 2: Example of the construction of one table

\begin{tabular}{ccccc}
\hline$N, \mathrm{kN}$ & $F_{0}, \mathrm{kN}$ & $M_{0}, \mathrm{Nm}$ & $a, \mathrm{~m}^{1 / 2}$ & $m, \mathrm{~m}$ \\
\hline 4.48 & 1.34 & 72.25 & 1.307 & 1.724 \\
\hline
\end{tabular}

Let us introduce the following dimensionless variables for the dry friction model:

$$
v=v / u, \quad \psi=v^{-1}=u / v .
$$


Taking into account the formulae for the longitudinal dry friction force, lateral dry friction force, and the dry friction torque (5), and substituting into (5) the dimensionless variables (12), we obtain the one-dimensional dependencies for dry friction forces and torque [19]:

$$
F_{\|} \quad{ }^{\Gamma} \psi\left(\psi^{2}+a\right)^{-1 / 2}, \quad F_{\perp}=k_{x} M_{0} \mathrm{v}\left(4 \mathrm{v}^{2}+m\right)^{-1 / 2}, \quad M_{C}=M_{0} \mathrm{v}\left(\mathrm{v}^{2}+m\right)^{-1 / 2} .
$$

Given $F_{0}, M_{0}, k_{x}, a$ and $m$ (see Table 2), we obtain the diagrams for the longitudinal dry friction force, lateral dry friction force, and the dry friction torque (13) (see Figures 3-5):
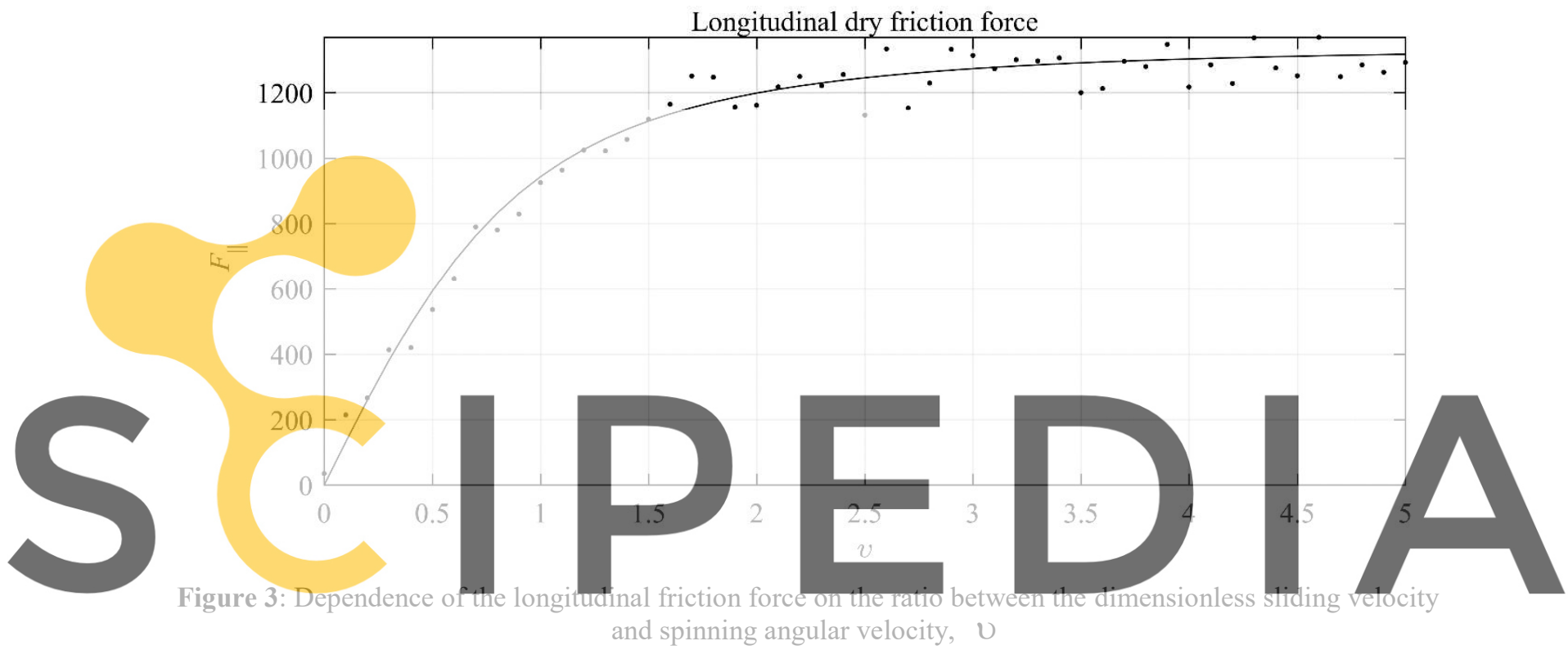

Register for free at https//www.scipedia.com to download the version without the watermark

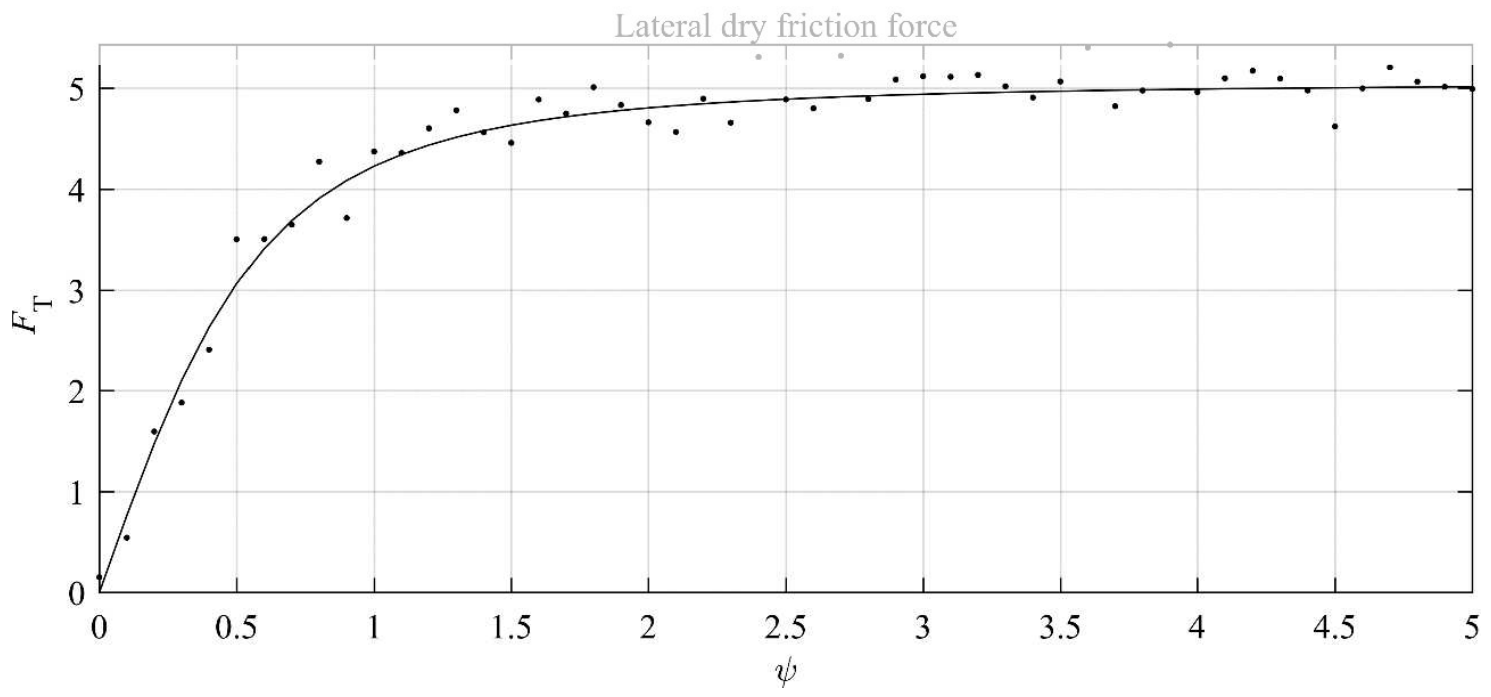

Figure 4: Dependence of the lateral friction force on the ratio between the spinning angular velocity and dimensionless sliding velocity, $\psi$. 


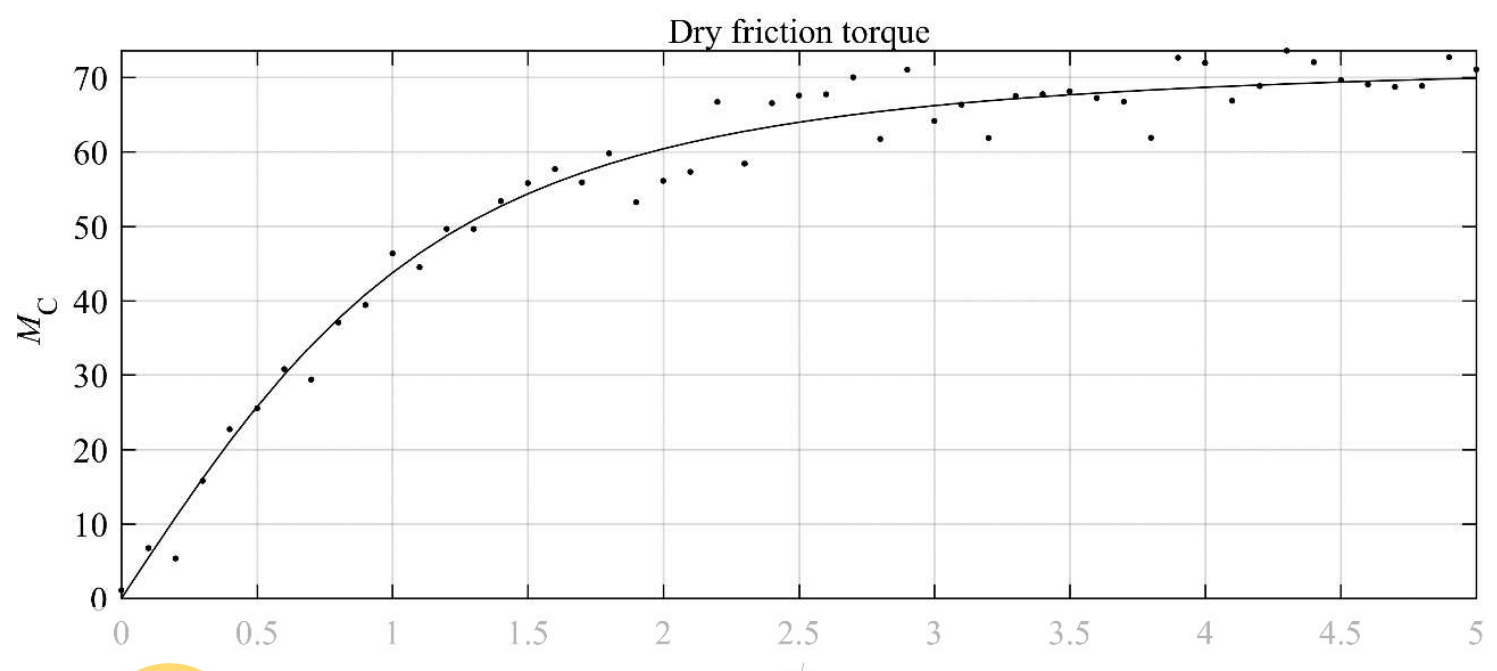

Figure 4: Dependence of the friction torque on the ratio between the spinning angular velocity and dimensionless sliding velocity, $\psi$.

Let us consider hence these diagrams $F_{\|}{ }^{\cdots}, F_{\perp}(\psi)$, and $M_{C}(\psi)$ as measured ones; thus,

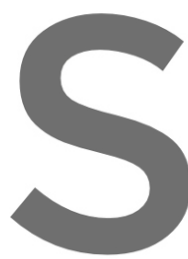
the aforementioned numerical optimization curves. The appropriat distribution with ampli shown above on Figures 2-4 by dotted lines.
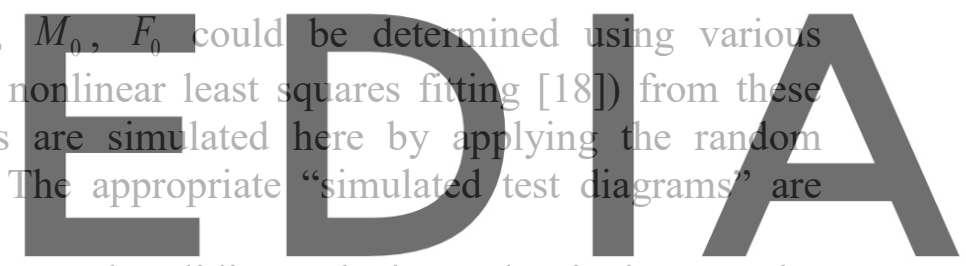

Thus, denoting the values of ratios between the sliding velocity and spinning angular

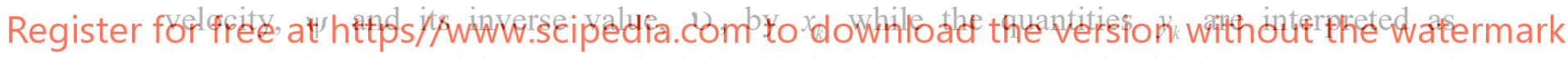
"measured" values of the longitudinal dry friction force $F_{n}$, lateral dry friction force $F_{\perp}$, and dry friction torque $M_{C}$ (Figures 3-5), we have the following condition [19]:

$$
\min \sum_{k=1}^{N}\left\|f\left(x_{k}\right)-y_{k}^{2}\right\|
$$

that leads to the estimates of the model factors $a, m, M_{0}, F_{0}$; the dry friction coefficient can be obtained as follows:

$$
f=\frac{F_{0}}{N} .
$$

The fitting (14) of the simulated test data results in the following values of the factors of the proposed coupled dry friction model obtained with $95 \%$ confidence bounds (see Table 3 ). The results shown in the Table 3 show a good correlation between the theoretical values and the ones obtained after the simulated experimental tests. 
Alexey A. Kireenkov, Sergey I. Zhavoronok

Table 3: Defined coefficients of the coupled dry friction model

\begin{tabular}{cccrl}
\hline Coefficient & Theoretical value & "Experimental" value & \multicolumn{2}{c}{ Confidence bounds } \\
\hline$f$ & 0.3 & 0.301 & $0.2958, \quad 0.3061$ \\
\hline$F_{0}$ & 1344 & 1348 & $1325, \quad 1371$ \\
\hline$M_{0}$ & 72.25 & 72.63 & $71,19, \quad 74.07$ \\
\hline$a$ & 1.030 & 1.024 & $0.8738,1.1740$ \\
\hline$m$ & 1.720 & 1.609 & 1.371, & 1.847 \\
\hline$k_{x}$ & 0.140 & 0.139 & 0.1376, & 0.1404 \\
\hline
\end{tabular}

Let us note that the simplest model of the contact interaction of the tire with the road combined with the Amonton-Coulomb dry friction model cannot define neither the lateral force nor the dry friction torque, moreover it overestimates the dry friction force value:

$$
F_{0} \approx \pi R^{2} p^{\star}, \quad . .427 \mathrm{kN} \text {. }
$$

\section{CONCLUSIONS}

The new model of the dry friction with coupled kinematics of the relative motion of

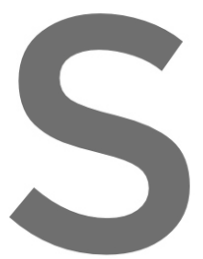

interacting solic

The real pneun

finite element

The trigonome

proposed, and
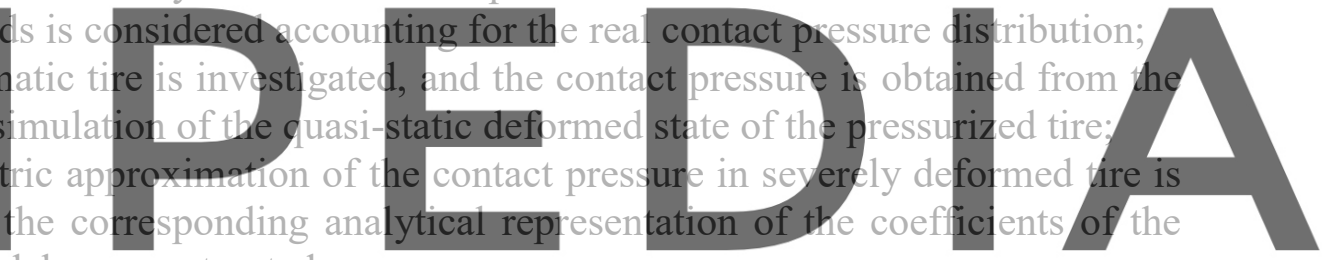

dry friction model are constructed

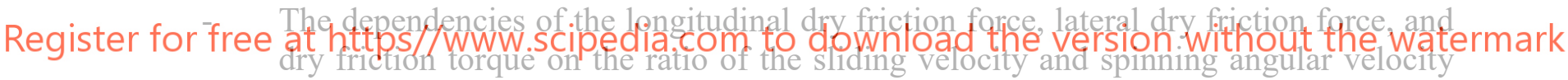

are constructed using dimensionless variables;

- $\quad$ The method of identification of the coefficients of the developed dry friction model using the experimental dependencies of the longitudinal dry friction force, lateral dry friction force, and the dry friction torque on the ratio of dimensionless sliding velocity and spinning angular velocity is proposed;

- $\quad$ The experimental data are simulated by adding the random noise to the theoretically computed diagrams, and the model coefficients are obtained from these perturbed diagrams by nonlinear least squares procedure;

- The rolling friction coefficient as well as other factors of the coupled dry friction model based on fraction-rational approximations are computed using the numerical simulation of the steady rolling of the tire corresponding to the possible test data;

- The good correlation between the exact model coefficients and the ones obtained from the simulated test curves is shown, and the principal possibility of identification of the model parameters after typical tests for a rolling tire is shown.

- The further development of the proposed approach may consist in the accounting of both vertical and lateral deformations of the tire as it was proposed in [21]. 


\section{ACKNOWLEDGEMENTS}

These investigations were supported by the RFBR, grant 20-08-01120, and partly by the state program of the scientific research works on the sections No. AAAA-A20120011690138-6 (Kireenkov A.A.) and No. AAAA-A19-119012290118-3 (Zhavoronok S.I.).

\section{REFERENCES}

[1] Keldysh, M.V. Selected Works, Mechanics. Nauka, Moscow (1985).

[2] Paceika, H.B. Tyre and vehicle dynamics. Elsevier (2006).

[3] Zagordan, A.A. and Zhavoronok, S. I. Main landing gear shimmy investigation using dry friction multicomponent model (in russian). Nelineiny Mir (2011) 10:646-56.

[4] Kireenkov, A.A. Improved theory of the combined dry friction in problems of aviation pneumatics dynamics. Proceedings of the 7th International Conference on Coupled Problems in Science and Engineering, COUPLED PROBLEMS 2017 (2017):1293-1298.

[5] Kireenkov, A.A. Improved friction model of the aviation tyre contact with the landing strip. IFAC-PapersOnLine (2018) 51(2):890-894. (DOI 10.1016/j.ifacol.2018.04.027)

[6] Kireenkov, A.A., Nushtaev, D.V. and Zhavoronok, S.I. A new approximate model of tyre accounting for both deformed state and dry friction forces in the contact spot on the background of the coupled model. MATEC Web of Conferences (2018) 211(08003). (DOI 10.1051/matecconf/201821108003)
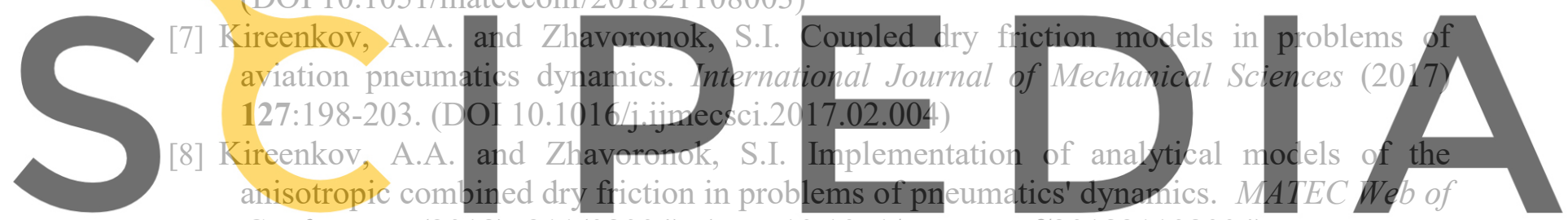

Conferences (2018) 211(08004). (DOI 10.1051/matecconf/201821108004)

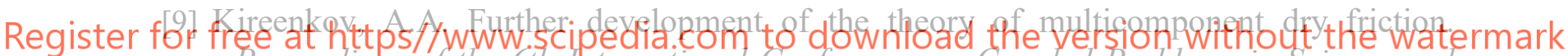
Proceedings of the Sth International Conference on Coupled Problems in Science and Engineering, COUPLED PROBLEMS 2015 (2015):203-209.

[10] Kireenkov, A.A. Three-dimensional model of combined dry friction and its application in non-holonomic mechanics. Proceedings of ENOC-2005, Eindhoven, Netherlands, August 7-12 (2005):571-577.

[11] Ramodanov, S.M. and Kireenkov, A.A. Controllability of a rigid body in a perfect fluid in the presence of friction. Proceedings of the 7th International Conference on Coupled Problems in Science and Engineering, COUPLED PROBLEMS 2017 (2017):179 - 184.

[12] Semendyaev, S.V. Solid system with two massive eccentrics on a rough plane: rotational case. IFAC-PapersOnLine (2018) 51 (2):884-889.

[13] Zhavoronok, S.I. and Kireenkov, A.A. On the effect of the anisotropic dry friction and the deformed state of tires on the shimmy initiation. Proceedings of the 7th International Conference on Coupled Problems in Science and Engineering, COUPLED PROBLEMS 2017 (2017):216-226.

[14] Kireenkov A.A. and Zhavoronok S.I. Anisotropic Combined Dry Friction in Problems of Pneumatics' Dynamics, Journal of Vibrational Engineering and Technologies (2020), 8 (2): 365-372. (DOI: 10.1007/s42417-019-00140-1). 
[15] Kireenkov A.A. and Ramodanov S.M. Combined Dry Friction Models in the Case of Random Distribution of the Normal Contact Stresses Inside of Contact Patches, Proceedings of the 8th International Conference on Coupled Problems in Science and Engineering, COUPLED PROBLEMS 2019 (2019):176-182.

[16] Bogoslovskii, S.E. and Kurdyumov, N. N. Numerical solution a problem of contact pneumatic truck tire with road surface. Proc TSU Tech Sci (2015) 8:138-47.

[17] Amosov, A.A., Zhavoronok, S.I. and Leont'iev, K.A. On the solution of some problems about stress-strain atate of thick anisotropic shells of revolution in three-dimensional problem statement. The J. of Mekhanika kompozitsionnykh materialov i konstruktsii (2004) 10(3):301-310 .

[18] Zhavoronok, S.I. The generalized Lagrange equations of the second kind for the extended three-dimensional n'th order theory of anisotropic shells. The J. of Mekhanika kompozitsionnykh materialov i konstruktsii (2015) 21(3):370-381.

[19] Kireenkov, A.A. and Zhavoronok S.I. Numeric-Analytical Methods of the Coefficients Definition of the Rolling Friction Model of the Pneumatic Aviation Tire, Proceedings of the 8th International Conference on Coupled Problems in Science and Engineering, COUPLED PROBLEMS 2019 (2019):204-212.

[20] Strutz, T. Data Fitting and Uncertainty (A practical introduction to weighted least squares and beyond). Springer Verlag, 2nd edition (2016).

[21] Kireenkov A.A., Zhavoronok S.I. and Nushtaev D.V. On tire models accounting for both deformed state and coupled dry friction in a contact spot. Computer Research and Modeling (2021) 13(1):163-173 (DOI: 10.20537/2076-7633-2021-13-1-163-173) 